\title{
Young's Modulus Measurements of Human Liver and Correlation with Pathological Findings
}

\author{
Wen-Chun Yeh, Yung-Ming Jeng ${ }^{1}$, Hey-Chi Hsu ${ }^{1}$, Po-Ling Kuo ${ }^{2}$, Meng-Lin Li, Pei-Ming Yang ${ }^{3}$ and Po \\ Huang Lee ${ }^{4}$ and Pai-Chi Li \\ Department of Electrical Engineering, National Taiwan University, \\ ${ }^{I}$ Department of Pathology and ${ }^{2}$ Department of Physical Medicine and Rehabilitation, ${ }^{3}$ Department of \\ Internal medicine and ${ }^{4}$ Department of Surgery, National Taiwan University Hospital, Taipei, Taiwan,
} R.O.C.

\begin{abstract}
Viral hepatitis causes fibrosis in liver and may change liver's mechanical properties. To evaluate the impact of fibrosis on elastic properties of human liver and to investigate potential benefits of ultrasonic elasticity imaging, nineteen fresh human liver samples and one hepatic tumor (focal nodular hyperplasia) sample obtained during operations were studied. Simple $1 \mathrm{D}$ estimates based on the cyclic compressionrelaxation method were preformed. Young's modulus values were derived from the predetermined strain (controlled by a step motor system) and the stress values (measured by an electronic balance). Each specimen subsequently received histological examination and a grade of liver fibrosis was scored from 0 to 5 . Results show that the Young's modulus values were at the order of several hundreds to thousands Pascals. The Young's modulus generally increased with the fibrosis grade though some discrepancies existed at the middle grades of fibrosis (score 1-3). The correlation between the fibrosis score and the Young's modulus was significant $(\mathrm{P}<0.01)$ based on the statistical analysis using the Pearson correlation method. It was concluded that severity of fibrosis had a good correlation with stiffness of liver. Results also indicated that the elasticity imaging of liver may provide significant clinical values if the Young's modulus can be accurately measured.
\end{abstract}

\section{Introduction}

Viral hepatitis such as hepatitis B or C may cause chronic inflammation of liver and induce fibrosis in the hepatic tissue. The degree of fibrosis may range from fibrous expansion in portal area to cirrhosis. Cirrhosis may cause severe complications. In addition, the incidence rate of hepatocellular carcinoma is also higher in these patients. Thus, detecting cirrhosis and screening for malignancy in the patients with chronic hepatitis are clinically important. Physicians typically screen these patients by using conventional B-mode ultrasound. However, detection of early stage cirrhosis using B-mode ultrasound is limited [1]. On the other hand, cirrhotic livers have abundant fibrosis septum between the acini and have been known to be harder than the normal liver by palpation. Since ultrasonic elasticity imaging can visualize the tissue stiffness and can provide physicians with a virtual "finger" to probe the elasticity inside of the body, it may be used to provide benefits in cirrhosis detection.

Elasticity imaging directly displays the tissue stiffness. If the elastic modulus of normal liver, cirrhotic liver and hepatic tumors can be accurately measured, elasticity imaging has the potential to differentiate liver lesions of different natures.

Several research groups have studied the elastic properties of liver [2]-[5]. Wang et al. [3] combined an optical method with an indentation technique to investigate the biomechanical response of pig's hepatic tissue. Results showed that the liver was a viscoelastic material. Yamashita and Kubota [4] estimated the elastic properties of human liver by measuring the internal displacement and strain. Emelianov et al. [5] studied the elastic properties of hepatic hemangiomas and found that hemangiomas were harder than the background liver tissue. Chen et al.[2] studied the Young's modulus of bovine liver and muscle and compared them with the results from direct mechanical measurements. Despite the numerous research results, quantitative and direct measurements of elastic properties of fresh human liver tissues are still lacking. It is the primary purpose of this paper to measure the elastic modulus of fresh human liver samples from patients with various liver diseases. Results are also correlated with pathology.

\section{Materials and Methods}

Nineteen fresh human liver samples and one hepatic tumor (focal nodular hyperplasia) were included in this study. The liver samples were obtained from patients with liver disease during operations at National Taiwan University Hospital (NTUH).

For each patient, a triangular or cubic sample was obtained from the surgical specimen immediately after it was sent to the Department of Pathology of NTUH. Each sample was preserved with normal saline solution at $4^{\circ} \mathrm{C}$. 
and was transported to the Ultrasonic Imaging Laboratory of the Department of Electrical Engineering, National Taiwan University. Measurements were made within 48 hours from the operation.

The specimens were cut to $1 \mathrm{~cm}^{3}$ cubes by using a surgical blade. After cutting, all prepared samples were preserved in a normal saline solution for at least 1 minute before measurements were performed in order to prevent dehydration. The venous vascular structure was avoided during cutting. The weight for each cubic specimen was around $1.1 \mathrm{~g}$ measured by an electronic balance (OHAUS 1P12KS, Florham Park, NJ) and the height of each specimen was measured by a ruler with a minimum scale of $1 \mathrm{~mm}$.

The electronic balance had a readability of 0.1 $\mathrm{g}$ and was placed under the specimen to measure the applied load. Figure 1 shows that the experimental setup consisted of the electronic balance, an acrylic plate as the compressor positioned by a step motor system (Q-Sync, Hsin-Chu, Taiwan) and a personal computer for central control and data acquisition.

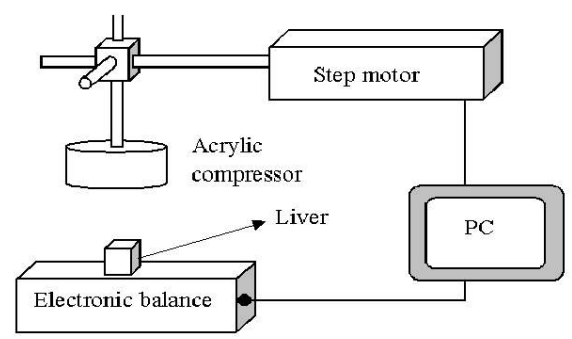

Fig. 1. Block diagram of the measurement setup.

Simple 1D estimates based on the cyclic compression-relaxation method was used [6]. For each sample, the compression procedures described below were used to determine the Young's modulus. The procedures were based on the incremental laws [7]. First, the sample was compressed sequentially to the three prespecified strain conditions (5\%,10\% and 15\%). At each strain, the sample was then cyclically compressed at an average compression rate of $7.1 \mathrm{~mm} / \mathrm{min}$ with a maximum compression of $500 \mu \mathrm{m}$. Each measurement composed of two consecutive compression-relaxation cycles and three measurements were made under each stain condition.

The Young's modulus for each specimen was computed from the slope of the stress-strain curve, where the strain was defined as the ratio of total deformation to the initial height. Since the compressor was positioned by the computercontrolled step-motor, strain at each position was readily obtained. For every compression, the maximum of consecutive readings from the electronic balance was obtained and divided by the area of contacting surface (i.e., $1 \mathrm{~cm}^{2}$ ) to determine the corresponding stress. Figure 2(a) shows typical readings from the electronic balance as a function of time. Each circle represents the maximum point for each compression step during the second cycle. The first cycle was used for pre-conditioning and the data were not used for Young's modulus estimation [7]. Figure 2(b) plots the selected maxima shown in Fig. 2(a) with the horizontal axis and vertical axis being converted to corresponding strain and stress, respectively. The solid line represents the best-fit line and the Young's modulus is determined by

the slope of the best-fit line. The average value from 3 measurements was calculated for each strain condition.

(a)

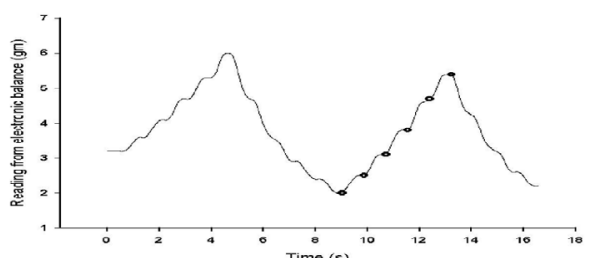

(b)

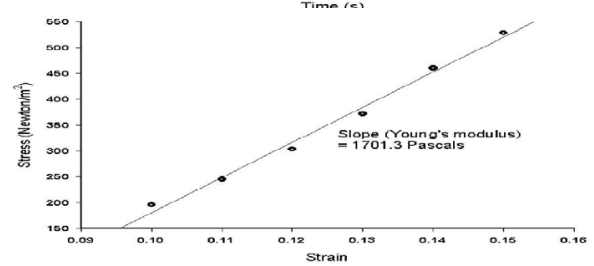

Fig. 2 Readings from the electronic balance as a function of time under a preload $10 \%$ strain. (a) Solid line represents the measurement curve and each circle corresponds to the maximum point at each step during compression in the second cycle. (b) Stress vs. strain. Solid line represents the best-fit line and the slope is the defined as the Young's modulus.

After the Young's modulus measurements were done, the specimens were subsequently fixed in formalin and embedded in paraffin. $5 \mu \mathrm{m}$ sections were cut and stained with hematoxylin and erosin for histological examinations. Fibrosis scores were assigned according to the criteria of the METAVIR Cooperative Study Group [8]. Liver fibrosis was scored from 0 to 4 according to the following definition: 0 : no fibrosis; 1: portal fibrosis without septa; 2: septa fibrosis; 3: numerous septa without cirrhosis and 4: cirrhosis. In cirrhotic livers, the widths of fibrotic bands varied and the score was further 
(a)

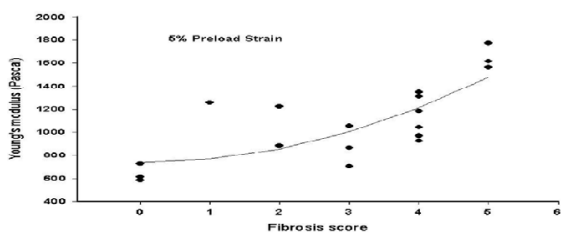

(b)

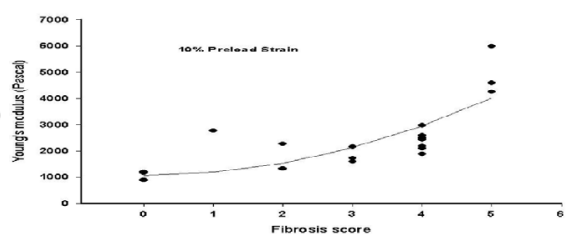

(c)

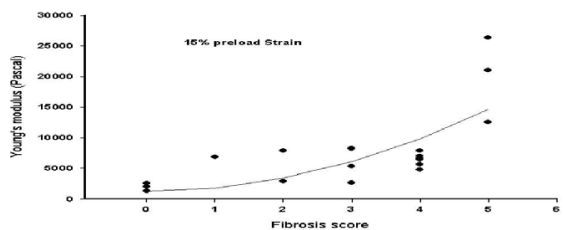

Fig. 3. Young's modulus vs. fibrosis score. Solid line represents regression curve under (a) 5\% preload strain, (b) $10 \%$ preload strain, and (c) $15 \%$ preload strain. The regression curves are non-linear and have a quadratic trend

divided such that a score of 4 represented thin fibrotic bands and a score of 5 was for thick fibrotic bands. The fatty change of the liver tissue was also evaluated. It was scored from 0 to 3 defined as the following: 0: no fatty change; 1: mild fatty change; 2 : moderate fatty change and 3: marked fatty change. The measured Young's modulus values were also correlated with the pathological findings using the Pearson correlation method.

\section{Results}

Table 1 shows that the mean value of the Young's modulus generally increased with the increased fibrosis score except for scores 1 and 2, The Young's modulus of severe fibrotic liver tissue (i.e., score 4 and 5) was much larger than that of normal tissue. Figures 3(a), 3(b) and 3(c) show distribution of the Young's modulus for different fibrosis scores under the preload strain conditions at $5 \%, 10 \%$ and $15 \%$, respectively. The horizontal axis is the fibrosis score and the vertical axis is the Young's modulus. The solid lines are best-fit curves based on the non-linear regression method. The best-fit curves in these figures have a strong quadratic trend. Figure 4 shows that the Young's modulus of the samples with fibrosis scores of 0,4 and 5 increased approximately exponentially with the preload strain. Obvious fibrosis bands around the acini were found in the tumor tissue (focal nodular hyperplasia) during the histological examination and the Young's modulus obtained from the tumor sample was within the range of those of the samples with a score of 4 .

Table 1: Average Young's modulus.

\begin{tabular}{|l|l|l|l|l|}
\hline \multirow{2}{*}{$\begin{array}{l}\text { Fibrosis } \\
\text { score }\end{array}$} & \multirow{2}{*}{$\begin{array}{l}\text { No of } \\
\text { specimen }\end{array}$} & & \multicolumn{3}{|l|}{ Young's modulus under Preload Strain } \\
\cline { 3 - 5 } & & $5 \%$ & $10 \%$ & $15 \%$ \\
\hline 0 & 3 & 642.55 & 1083.55 & 2000.51 \\
\hline 1 & 1 & 1257.34 & 2767.52 & 6809.04 \\
\hline 2 & 2 & 1053.50 & 1788.50 & 5404.21 \\
\hline 3 & 3 & 874.81 & 1820.51 & 5389.35 \\
\hline 4 & 7 & 1106.42 & 2373.56 & 6272.28 \\
\hline 5 & 3 & 1649.01 & 4930.71 & 19983.18 \\
\hline
\end{tabular}

The Pearson correlation method was also used to evaluate the correlation between the fibrosis score and the value of Young's modulus. The Pearson correlation coefficients $(r)$ were listed in Table 2. The $r$ between the fibrosis score and the value of Young's modulus under $5 \%, 10 \%$ and $15 \%$ preload strain conditions were $0.702,0.669$ and 0.626 . These values indicated that positive correlation existed and the correlation was significant at the 0.01 level $(\mathrm{P}<0.01)$. The correlation between the Young's modulus measured at different preload strains was all higher than 0.850 . There was no obvious correlation between the fatty change score and the Young's modulus.

Table 2: The Pearson correlation between the fibrosis score /fatty change score and the Young's modulus.

\begin{tabular}{l|l|l|l|l|l}
\hline & $\begin{array}{l}\text { Fibrosis } \\
\text { score }\end{array}$ & $\begin{array}{l}\text { Fatty } \\
\text { change } \\
\text { score }\end{array}$ & YM (5\%PS) & YM (10\%PS) & YM (15\%PS) \\
\hline Fibrosis score & 1 & 0.162 & $0.702^{* * *}$ & $0.669^{* * *}$ & $0.626^{* * *}$ \\
\hline Fatty change score & 0.162 & 1 & 0.344 & 0.251 & 0.270 \\
\hline YM ( 5\%PS) & $0.702^{* *}$ & 0.344 & 1 & $0.912^{* * *}$ & $0.856^{* * *}$ \\
\hline YM (10\%PS) & $0.669^{* * *}$ & 0.251 & $0.912^{* * *}$ & 1 & $0.958^{* *}$ \\
\hline YM (15\%PS) & $0.626^{* * *}$ & 0.270 & $0.856^{* * *}$ & $0.958^{* * *}$ & 1 \\
\hline
\end{tabular}

YM: Young's modulus; PS: preload strain

\section{Discussion and Conclusions}

This paper investigated the relationship between the fibrosis grade and the Young's modulus. The Young's modulus was directly measured. Results showed that severity of fibrosis had a good correlation with the Young's modulus. The Young's modulus of fresh human liver at $5 \%$ preload strain ranged from 586 to 1733 Pascals at different fibrosis stages. The results are similar to the Young's modulus measurements of bovine liver under 1-5\% strain [2]. The mean values of Young's modulus in Table 1 generally increased with increasing fibrosis grades except for scores 1 and 2. In other words, the cirrhotic tissue (scores 4 and 5) is 
harder than normal tissue (score 0). Yamashita and Kubota [4] estimated elasticity of soft tissue by the measurement of internal displacement and strain. It was also found that the normal liver tissue had a relatively larger local displacement than the cirrhosis of liver, (i.e., cirrhotic liver was stiffer than normal liver). Such a finding is also consistent with the results shown in this paper.

The regression curves between the fibrosis score and the Young's modulus generally had a quadratic trend as shown in Fig. 3. Discrepancies existed in some samples with fibrosis scores of 1-2. This may be due to the small number of samples for fibrosis scores of 1 and 2 , measurement errors, and other changes in the liver tissue that may affect liver stiffness. For example, the fatty change of liver fills some of the liver cells with triglyceride and may alter the mechanical properties of liver. In our study, the non-cirrhotic liver samples with fatty change seemed harder than the tissue with less fatty change at the same fibrosis score (fibrosis scores 2 and 3). However, no statistical significance was found as shown in Table 2. Further studies are required to explore other causes that can alter the stiffness of liver.

Fresh liver samples were used in this study without formalin fixation. However, there were some difficulties in cutting the fresh liver samples. The cutting surface of liver was difficult to be very smooth especially for soft normal liver samples. To reduce the measurement errors caused by uneven surface and to ensure a good contact between the liver sample and the compressor, the starting preload strain was $5 \%$ instead of the $0 \%$ strain used by Kuo et al.[6] for tendon stiffness measurements. Nevertheless, errors may exist in measuring the height of the specimen. The height of the specimen was cut to be $10 \mathrm{~mm}$ measured by a ruler with a minimum scale of $1 \mathrm{~mm}$. Thus, the actual height may have ranged between $9.5 \mathrm{~mm}$ and $10.5 \mathrm{~mm}$. Taking the potential measurement error into account, the actual preload strains ranged from $4.76 \%-5.26 \%, 9.52 \%-10.53 \%$ and $14.29 \%-15.79 \%$ for the three pre-specified preload strain at $5 \%, 10 \%$ and $15 \%$, respectively. Influence of the measurement error is shown in Fig. 4. The actual ranges of preload strains are shown as the vertical dashed lines. Values of Young's modulus at the highest (15\%) preload strain vary the most due to the fact that the Young's modulus increased nonlinearly with the applied strain. Nevertheless, distinction between the normal and the cirrhotic samples is still obvious.

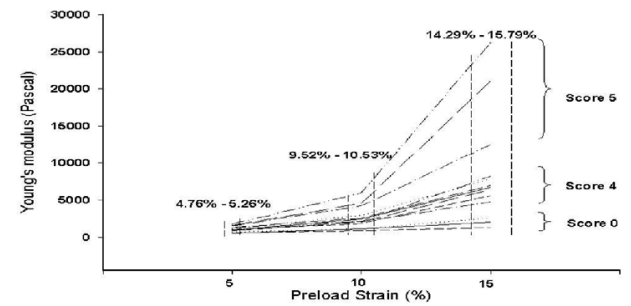

Fig. 4: The Young's modulus - strain curves : the range of errors and the strain hardening effect.

Chen et al. [2] found that the stress-strain curve becomes noticeably nonlinear when tissue strain exceeded $10 \%$. As the load further increases, the stress-strain curve becomes exponential. The nonlinearity is also known as the strain hardening effect. The strain hardening effect is demonstrated in Fig. 4 in that the Young's modulus increases faster at higher strains. Such a phenomenon is present for all specimens including both liver tissue and tumor. Also note that the strain hardening effect is more apparent for stiffer tissues. The differences of the strain hardening effect among tissues may be utilized to enhance the contrast between tissues with different elastic properties.

\section{References}

[1] Yang PM, Huang GT, Lin JT et al. Ultrasonography in the diagnosis of benign diffuse parenchymal liver disease: a prospective study. J Formosan Med Assoc 187(10):966-977,.1988.

[2] Chen EJ, Norvokovski J, Jenkins WK, O'brien, Jr WD. Young's modulus measurements of soft tissues with application to elasticity imaging. IEEE Trans Ultrason Ferroelect Freq Contr 43(1):191-194,1996.

[3] Wang BC, Wang GR, Yan DH, Liu YP. An experimental study on biomechanical properties of hepatic tissue using a new measuring method. BioMed Mat Eng 2(3):133138, 1992.

[4] Yamashita Y, Kubota M. Ultrasonic characterization of tissue hardness in the in vivo human liver. IEEE Ultrason symp 1449-1453, 1994.

[5] Emelianov SY, Rubin JM, Lubinski MA, Skovoroda AR, O'Donnell M. Elastic imaging of liver: is a hemangioma hard or soft. IEEE Ultrason symp 1749-1752, 1998.

[6] Kuo PL, Li PC, Li ML. Elastic properties of tendon measured by two different Approaches. Ultrasound Med Biol 2001 (in press).

[7] Fung YC. Biomechanics: mechanical properties of living tissues. New York: Springer-Vela, 1259263, 1993.

[8] Poynard T, Bedossa P, Opolon P. Natural history of liver fibrosis progression in patients with chronic hepatitis C. The OBSVIRC, METAVIR, CLINIVIR, and DOSVIRC groups. Lancet 349:825-832, 1997. 